\title{
Axions and the luminosity function of white dwarfs. The thin and thick disks, and the halo
}

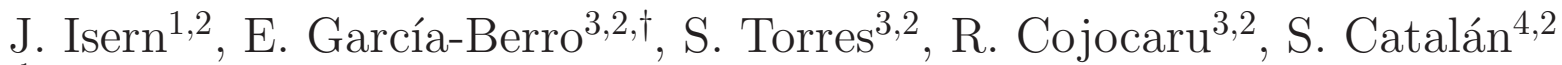 \\ ${ }^{1}$ Institute of Space Sciences (ICE, CSIC), Campus UAB, Cami de Can Magrans s/n, 08193 Cerdanyola del Vallès (Barcelona), Spain \\ 2 Institute for Space Studies of Catalonia, c/Gran Capità 2-4, Edif. Nexus 201, 08034 Barcelona, Spain \\ ${ }^{3}$ Departament de Física, Universitat Politècnica de Catalunya, c/Esteve Terrades 5, 08860 Castelldefels, Spain \\ 4 Center for Astronomy, National University of Ireland, Newcastle Road, SW4 794 Galway, Ireland \\ $\dagger$ Deceased September 23rd, 2017
}

Accepted .... Received 2017; in original form 2017

\begin{abstract}
The evolution of white dwarfs is a simple gravothermal process of cooling. Since the shape of their luminosity function is sensitive to the characteristic cooling time, it is possible to use its slope to test the existence of additional sources or sinks of energy, such as those predicted by alternative physical theories. The aim of this paper is to study if the changes in the slope of the white dwarf luminosity function around bolometric magnitudes ranging from 8 to 10 and previously attributed to axion emission are, effectively, a consequence of the existence of axions and not an artifact introduced by the star formation rate. We compute theoretical luminosity functions of the thin and thick disk, and of the stellar halo including axion emission and we compare them with the existing observed luminosity functions. Since these stellar populations have different star formation histories, the slope change should be present in all of them at the same place if it is due to axions or any other intrinsic cooling mechanism. The signature of an unexpected cooling seems to be present in the luminosity functions of the thin and thick disks, as well as in the halo luminosity function. This additional cooling is compatible with axion emission, thus supporting to the idea that DFSZ axions, with a mass in the range of 4 to $10 \mathrm{meV}$, could exist. If this were the case, these axions could be detected by the future solar axioscope IAXO.
\end{abstract}

Key words: axions - stars: white dwarfs - galaxy: luminosity function, thin, thick disc, halo

\section{INTRODUCTION}

White dwarfs are the final evolutionary stage of low and intermediate-mass stars - namely, those with $M \leqslant 10 \pm$ $2 M_{\odot}$ (Althaus et al. 2010). They are degenerate objects and, consequently, they cannot obtain energy from thermonuclear reactions and their evolution is just a gravothermal process of cooling. Their structure is relatively simple. They have a degenerate core that contains the bulk of mass and acts as a reservoir of energy, and a partially degenerate envelope that controls the energy outflow. White dwarfs with masses $M \lesssim 0.4 M_{\odot}$ have He cores, while those with a $M \gtrsim 1.1 M_{\odot}$ have O-Ne cores. Finally, those with intermediate masses, which represent the vast majority, have a core made of a mixture of $\mathrm{C}$ and $\mathrm{O}$ plus some impurities like ${ }^{22} \mathrm{Ne}$. All of them are surrounded by a thin He layer with a mass $\sim 10^{-2} M_{\mathrm{WD}}$. However, in $\sim 75 \%$ of the cases, these stars are also surrounded by an even thinner layer of hydrogen with a mass between $10^{-4}$ and $10^{-15} M_{\odot}$. White dwarfs displaying hydrogen in their spectra are known as DA and the remaining ones are generically referred to as non-DA.

Simple theoretical considerations show that the coolest white dwarfs have ages $\gtrsim 10$ Gyr. Therefore, they allow to obtain important information about the past history of the Galaxy, in particular about the age of the disk, the halo and the system of globular and open clusters, as well as the star formation rate - see the reviews by Koester \& Chanmugam (1990); Isern et al. (1998a); Fontaine et al. (2001); Koester (2002); Hansen \& Liebert (2003); Hansen (2004); Althaus et al. (2010); Isern et al. (2013); García-Berro \& Oswalt (2016). Moreover, it is important to realize that the accuracy and reliability of the formation history of all these stellar components will be much improved when the data obtained by Gaia will become available (Barstow et al. 2014).

The tool to obtain such information is the luminosity function, i.e. the distribution of white dwarfs as a function of their luminosity (see Fig. 1). This function was de- 
rived for the first time by Weidemann (1968), about four decades ago, and was improved by several authors during the nineties (Liebert et al. 1988; Evans 1992; Oswalt et al. 1996; Leggett et al. 1998; Knox et al. 1999). These early luminosity functions proved that the evolution of white dwarfs is just a cooling process and that there was a cut-off in the distribution caused by the finite age of the Galaxy.

The position of the cut-off is sensitive to the cooling rate and, consequently, it can be used to constrain any new theory or hypothesis implying the introduction of an additional source or sink of energy. However the low number of stars in the samples, few hundreds, and the uncertainties in the position of the cut-off prevented anything else than obtaining upper bounds. This was the cases of the mass of the axion (Raffelt 1996) or the secular drift of the Newton constant (Garcia-Berro et al. 1995), just to cite two examples.

The advent of large cosmological surveys like the Sloan Digital Sky Survey (SDSS) introduced a noticeable improvement in the precision and accuracy of the luminosity function of white dwarfs since they allowed to increase the sample size to several thousands of stars. Fig. 1 shows the luminosity function of disk white dwarfs obtained by several authors. Black solid squares represent the data corresponding to $\sim 6000 \mathrm{DA}$ and non-DA white dwarfs obtained from the SDSS Data Release 3 and the USNO-B catalogues (Harris et al. 2006) using the proper motion method. A weak point of the luminosity functions obtained from the SDSS catalogues is that the integration time is fixed and dim white dwarfs systematically have poorer $\mathrm{S} / \mathrm{N}$ ratios (Limoges \& Bergeron 2010). Fortunately, the Super COSMOS Sky Survey, a completely independent survey that also uses proper motion to identify white dwarfs, is completely free of this systematic effect. The luminosity function obtained from this catalogue is represented by magenta stars (Rowell \& Hambly 2011) in Fig. 1. As can be seen, both functions nearly coincide over a large part of the brightness interval when normalized to $\log \left(L / L_{\odot}\right) \approx-3$ or $M_{\mathrm{bol}} \approx 12$. At large brightness, $M_{\mathrm{bol}} \lesssim 6$, both luminosity functions display a large dispersion, not plotted in the figure, as a consequence of the fact that the proper motion method is not appropriate there. One way to circumvent this problem is using the UV-excess technique (Krzesinski et al. 2009). The results obtained with this technique are represented by black crosses in Fig. 1 after matching their dim region with the corresponding bright segment of the Harris et al. (2006) distribution. As a complement, the luminosity function of the dimmest white dwarfs obtained by Leggett et al. (1998) has also been included in Fig. 1 as red triangles. The discrepancies at low luminosities are due to the difficulty to separate DAs from non-DAs and to the different behavior of the envelope. The green crosses represent the luminosity function recently obtained by Munn et al. (2017), also from the SDSS, that represents a noticeable improvement of the data obtained by Harris et al. (2006). Finally, the blue hollow squares represent the luminosity function of only DA stars (DeGennaro et al. 2008) normalized to the other luminosity functions at $\log \left(L / L_{\odot}\right)=-3$ in order to compare the slopes.

The improved quality of the new luminosity functions has allowed, for the first time, to determine their shape and to use the slope as a tool to test new physical theories (Raffelt 1996; Isern \& García-Berro 2008). If an additional

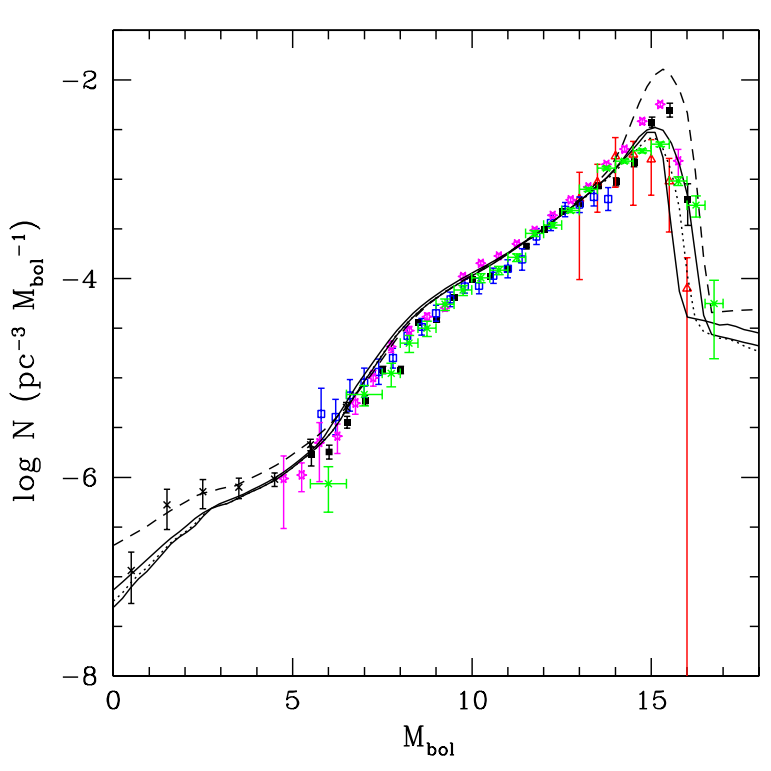

Figure 1. Luminosity functions obtained from different authors: black solid squares (Harris et al. 2006), open blue squares (DeGennaro et al. 2008), black crosses (Krzesinski et al. 2009), magenta stars (Rowell \& Hambly 2011) and green stars (Munn et al. 2017). The two solid black lines represent the theoretical luminosity functions obtained assuming a constant SFR and an age of the Galaxy of 10 and 13 Gyr, respectively. The dashed line represents the one obtained with a decreasing exponential SFR and, finally, the dotted line is obtained using an almost constant SFR with an exponential tail. See text for further details.

source or sink of energy is added to the energy budget of the white dwarf, the characteristic cooling time is modified and its imprint appears in the luminosity function, as can be seen in Fig. 1, where the change of slope that appears at magnitudes $\sim 8$ is caused by the transition from the cooling dominated by neutrinos to the one dominated by photons. As an example, this technique was used by Isern et al. (2008) to suggest that axions of the DFSZ type could be contributing to the cooling of white dwarfs.

One of the main uncertainties comes from the star formation rate (SFR). If this function is relatively smooth and does not increase abruptly with time, the shape of the bright branch of the luminosity function is entirely determined by the physical properties of white dwarf stars (Isern \& García-Berro 2008). It has been shown (Rowell 2013) that, by adjusting bin by bin it is possible to find a SFR that almost completely fits the observed WDLF and is compatible with SFRs obtained with independent methods (Cignoni et al. 2006).

This SFR, however, has been obtained as an "ad hoc" fit to the observations, employing the existing white dwarf models. Consequently, other models with different physical ingredients would provide different solutions. One way to break this degeneracy and to decide if it is necessary to include new physics is to examine the luminosity functions of populations that have different stellar formation histories. In this case, if axions (or any other physical effect) are responsible for this anomalous cooling, their signature would be 
present in all the luminosity functions at roughly the same luminosities.

Another important uncertainty comes from the adopted white dwarf scale height at the moment of estimating their density. Harris et al. (2006) showed that changing the scale height has a noticeable effect on the shape of the bright branch of their luminosity function. Since the Munn et al. (2017) luminosity function is representative of a mixture of thin and thick disk stars, Kilic et al. (2017) have also shown that it is possible to remove the mentioned discrepancy between the observational and theoretical luminosity functions by just adopting a variable scale height connecting the thin and thick disk populations. This argument, that is correct, reinforces the convenience of analyzing the luminosity function of white dwarfs belonging to populations with independent scale heights and with different star formation histories to disentangle the intrinsic and group properties.

One possibility is provided by the ordinary galactic populations. The common picture is that spiral galaxies form inside a spinning dark matter halo. In the first stage, the gas collapses in a dynamical time scale of $\sim 10^{8}$ yrs, leaving behind a stellar halo, and settles down into a disk from where the stars form. Galactic disks can be easily destroyed by mergers with other structures of similar mass. Therefore, the existence of an undamaged disk implies that it has no suffered important mergers since it was born. Furthermore, if an early formed disk is perturbed by a minor merger, it can form a thick disk which, in turn, can lead to the formation of a thin disk inside it. According to this picture, the present structure of the Milky Way could be the result of the following sequence of events (Reid 2005): i) formation of a primitive halo at $t \sim 11-12$ Gyr; ii) episodes of minor mergers from the satellite galactic system; iii) formation of the disk at $t \sim 10-11 \mathrm{Gyr} ;$ iv) formation of a thick disc at $t \sim 9-10$ Gyr; v) formation of the thin disk at $t \sim 8$ Gyr. Therefore, it seems reasonable to assume that the SFR of the inner halo, and of the thin and thick disks are reasonably independent. Using kinematic arguments it is possible to distinguish between the white dwarfs that belong to these three structures (Rowell \& Hambly 2011; Munn et al. 2017) and, consequently it is natural to look for any anomalous cooling in the region $M_{\mathrm{bol}} \sim 7-12$ of the luminosity functions of these white dwarf populations. In particular, the axion case will be analysed as an example.

Another possibility is provided by globular clusters (Hansen et al. 2015; García-Berro et al. 2014; Goldsbury et al. 2016), but the analysis is not straightforward since globulars are not made of a single stellar population but of two or even three (Gratton et al. 2012). Furthermore, the dynamics of the cluster, sink of heavy stars towards the center for instance, can be strongly modified by the presence of moderately massive black holes in the center, as is the case of 47 Tuc (Kiziltan et al. 2017). For these reasons, we will analyze the case of globular clusters in a separate paper.

\section{THE LUMINOSITY FUNCTION OF WHITE DWARFS}

The white dwarf luminosity function is defined as the number density of white dwarfs of a given magnitude per unit

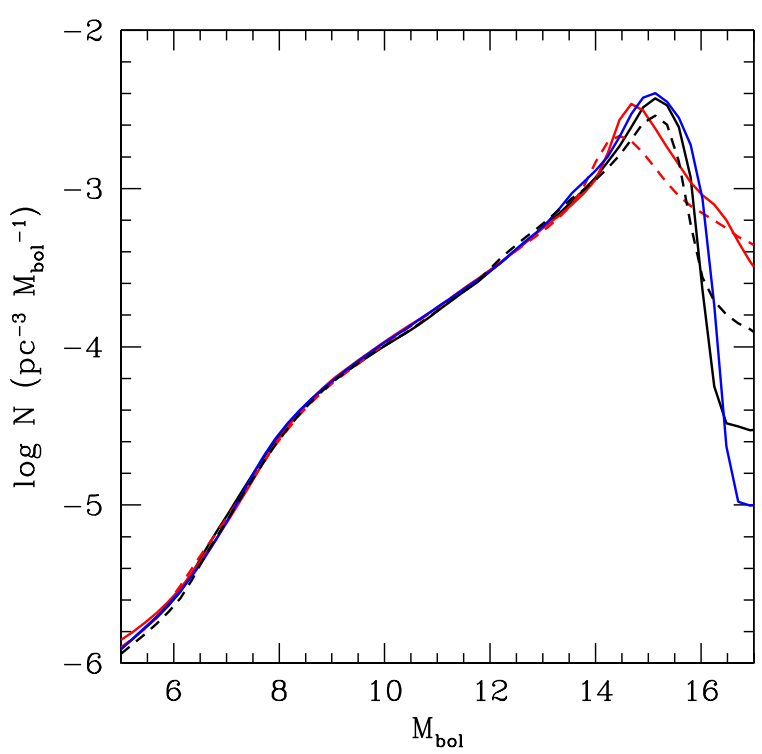

Figure 2. Theoretical luminosity functions obtained with the CO white dwarf models of: Salaris et al. (2010) - continuous, pure DA stars, and dashed, $80 \%$ DAs and 20\%non-DAs, black lines, Renedo et al. (2010) - blue line - and Fontaine et al. (2001) continuous, thick H-envelope, and dashed, thin H-envelope, red lines.

magnitude interval:

$$
n(l)=\int_{M_{\mathrm{l}}}^{M_{\mathrm{u}}} \Phi(M) \Psi(t) \tau_{\mathrm{cool}}(l, M) d M
$$

In this expression, $t$ satisfies the condition $t=T-$ $t_{\text {cool }}(l, M)-t_{\mathrm{PS}}(M)$, where $l=-\log \left(L / L_{\odot}\right), M$ is the mass of the parent star (for convenience all white dwarfs are labeled with the mass of the main sequence progenitor), $t_{\text {cool }}$ is the cooling time down to luminosity $l, \tau_{\text {cool }}=d t / d M_{\text {bol }}$ is the characteristic cooling time, $t_{\mathrm{PS}}$ is the lifetime of the progenitor of the white dwarf, and $T$ is the age of the population under study. $M_{\mathrm{u}}$ and $M_{1}$ are the maximum and the minimum masses of the main sequence stars able to produce a white dwarf of luminosity $l$. Therefore, $M_{1}$ satisfies the condition

$$
T=t_{\mathrm{cool}}\left(l, M_{1}\right)+t_{\mathrm{PS}}\left(M_{1}\right)
$$

The remaining quantities, the initial mass function, $\Phi(M)$, and the star formation rate, $\Psi(t)$, are not known a priori and depend on the properties of the stellar population under study. Since the total density of white dwarfs is not yet well determined, to compare theory and observations it is customary to normalize the white dwarf luminosity function to the bin with the smallest error bar, usually at $l \simeq 3$. Finally, although it does not appear explicitly in the previous expressions, it is necessary to provide a relationship relating the mass of the white dwarf with the mass of its progenitor. In summary, if the stellar properties are well known it is possible to obtain information about the galactic properties, specifically the star formation rate and the age of the Galaxy. Yet this is not the only possibility, since the bright branch of the white dwarf luminosity function - that with 
bolometric magnitude $M_{\text {bol }} \lesssim 13$ - is almost independent of the assumed star formation rate. This can be explained employing simple arguments. Eq. (1) can be written as:

$$
n(l)=\left\langle\tau_{\mathrm{cool}}\right\rangle \int_{M_{1}}^{M_{\mathrm{u}}} \phi(M) \psi\left(T-t_{\mathrm{cool}}-t_{\mathrm{PS}}\right) d M .
$$

Restricting ourselves to bright white dwarfs - namely, those for which $t_{\text {cool }}$ is small - the lower limit of the integral is satisfied by low-mass stars and, as a consequence of the strong dependence of the main sequence lifetimes with mass, it adopts a value that is almost independent of the luminosity under consideration. Therefore, if $\psi$ is a wellbehaved function and $T$ is large enough, the lower limit is almost independent of the luminosity, and the value of the integral is incorporated into the normalization constant in such a way that the shape of the luminosity function only depends on the averaged physical properties of the white dwarfs (Isern \& García-Berro 2008; Isern et al. 2009). This average is dominated by low mass white dwarfs and, as far as the mass spectrum is not strongly perturbed by the adopted star formation or the initial mass function, it is representative of the intrinsic properties of white dwarfs.

It is important to emphasize here that the luminosity band we are considering, $6 \lesssim M_{\text {bol }} \lesssim 13$ is the one with less theoretical uncertainties and where all the present models almost coincide (Hansen \& Liebert 2003). In particular, Fig. 2 shows, after normalization, the different luminosity functions obtained with the BaSTI models ${ }^{1}$ (Salaris et al. 2010), La Plata models ${ }^{2}$ (Renedo et al. 2010), and Montreal models ${ }^{3}$ (Fontaine et al. 2001). These luminosity functions were computed as in Isern et al. (1998b) and in Miller Bertolami et al. (2014), using the initial final mass relationship of Catalán et al. (2008).

As can be seen, these luminosity functions are almost indistinguishable in the region $M_{\mathrm{bol}} \lesssim 13$. The differences due to the models themselves or the shape of the SFR only appear in the regions containing cool or very bright white dwarfs. Unfortunately, the present observational uncertainties in these regions prevent at present to discriminate among the different possibilities. In the following we will adopt the BaSTI models as the reference for calculations. A discussion on the sensitivity of the luminosity function to the different inputs can be found in Isern et al. (2009) and in http://astro.uni-tuebingen.de/ rauch/EUROWD10.html

In the bright region considered here, the slope of the relationship between the luminosity and the core temperature of DA and non-DA white dwarfs almost coincide,

$1 \mathrm{C} / \mathrm{O}$ abundances stratified, $M_{\mathrm{He}}=10^{-2} M_{\mathrm{wd}}, M_{\mathrm{H}}=$ $10^{-4} M_{\mathrm{wd}}$ for DA models, and $M_{\mathrm{He}}=10^{-3.5} M_{\mathrm{wd}}$. Publicly available at: http://albione.oa-teramo.inaf.it

${ }^{2} \mathrm{C} / \mathrm{O}$ abundances stratified,masses of the envelope different for each mass of the white dwarf and in the range $10^{-2.486} \lesssim M_{\mathrm{He}} \lesssim 10^{-1.567} M_{\mathrm{wd}}, 10^{-486} \lesssim$ $M_{\mathrm{H}} \lesssim 10^{-3.441} M_{\mathrm{wd}}$ for DA models. Publicly available at http://www.fcaglp.unlp.edu.ar/ althaus/

$3 \mathrm{C} / \mathrm{O}$ half and half and uniformly distributed in the star. Thick envelopes $M_{\mathrm{He}}=10^{-2} M_{\mathrm{wd}}, M_{\mathrm{H}}=10^{-4} M_{\mathrm{wd}}$, and thin envelopes $M_{\mathrm{He}}=10^{-2} M_{\mathrm{wd}}, M_{\mathrm{H}}=10^{-10} M_{\mathrm{wd}}$. Publicly available at http://www. astro.umontreal.ca/ bergeron/CoolingModels

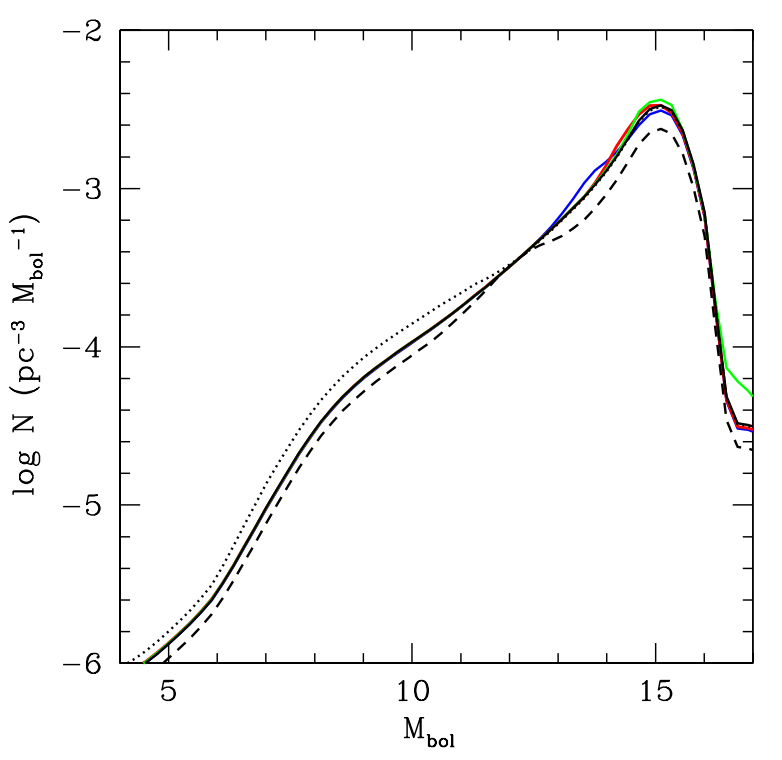

Figure 3. Signatures in the disk white dwarf luminosity function of different bursts of star formation. The solid lines show the luminosity function for a constant star formation rate (black line) and for a constant star formation rate with three bursts of star formation occurring 4, 6 and 8 Gyr ago (blue, red and green lines). The dotted and dashed lines correspond to a constant star formation rate with bursts occurring 1 and 2 Gyr ago, respectively. See text for additional details.

see Fig. 1 of Salaris et al. (2010). If we adopt a relationship of the form $L=g T_{\mathrm{c}}^{\gamma}$, where $\gamma=\gamma_{\mathrm{DA}} \approx \gamma_{\mathrm{nDA}}$ in the luminosity range mentioned before, and we neglect the compression term, $L \approx-d U / d t \approx-C_{\mathrm{V}} d T_{\mathrm{c}} / d t$, we obtain $n(l) \propto i^{-1}=-L(d L / d t)^{-1}=C_{\mathrm{V}}(g \gamma)^{-1} T_{C}^{1-\gamma}$, and the DA and non-DA luminosity functions almost overlap in this luminosity interval after normalization. This is the reason why the luminosity function of DeGennaro et al. (2008) containing only DAs coincides with those containing DAs and nonDAs after normalization.

Fig. 1 also displays the theoretical disk white dwarf luminosity functions obtained assuming a constant SFR but two ages of the Galaxy of 10 and 13 Gyr, respectively, a decreasing exponential SFR, $\Psi \propto \exp (-t / \tau), \tau=3 \mathrm{Gyr}$, and, finally, an almost constant SFR with an exponentially decreasing tail that represents models where the star formation propagated from the center to the periphery, $\Psi \propto\left(1+\exp \left[\left(t-t_{0}\right) / \tau\right]\right)^{-1}, \tau=3 \mathrm{Gyr}, t_{0}=10 \mathrm{Gyr}$. As can be seen, in the region $6 \lesssim M_{\mathrm{bol}} \lesssim 13$, all luminosity functions overlap as far as the SFR is smooth enough.

Now we go one step forward and we ask ourselves for the effects on the luminosity function of short bursts of star formation. This question is important as it may modify the slope of the luminosity function (Noh \& Scalo 1990). For instance, when a young burst is considered, low-mass main sequence stars have no time to become white dwarfs. Since $M_{1}$ in Eq. (3) depends on the luminosity, the slope of the luminosity function is affected. Fig. 3 represents the luminosity functions obtained when a burst of star formation is added to a constant SFR, $\psi(t)=\psi_{0}\left[1+\delta\left(t_{1}, \Delta t\right)\right]$. Here 
$\delta\left(t_{1}\right)=1$, if $t_{1}-\Delta t \leqslant t \leqslant t_{1}$, where $t_{1}$ is the time at which the burst started and $\Delta t$ is its duration of the burst, and zero otherwise. The dotted and the dashed lines represent the luminosity functions when a burst of duration $\Delta t=1$ Gyr at 1 and 2 Gyr, respectively. As can be seen, the effects of these bursts are clearly visible in the slope of the luminosity function. On the contrary, if the burst of star formation is old enough, the corresponding luminosity functions are barely modified. This is clearly borne out by inspecting the solid lines in Fig. 3, which represent the luminosity functions for bursts occurring at 4, 6, and $8 \mathrm{Gyr}$, respectively. It is evident that, since the old generations of stars are still producing new white dwarfs, a precise determination of the luminosity function at low luminsities could provide important constraints to the temporal evolution of the SFR.

\section{THE AXION CASE}

The most widely accepted solution to the strong CP problem of quantum chromodynamics consists in the introduction of a new symmetry, the Peccei-Quinn symmetry (Peccei \& Quinn 1977b,a), that spontaneously breaks at some energy scale and leads to the formation of a light pseudo-scalar particle named axion (Weinberg 1978; Wilczek 1978). This energy scale is not fixed by the theory, but it has to be much larger than the electroweak scale to ensure a coupling between axions and matter weak enough to account for the lack of detection up to now. The mass of axions and the energy scale are related by: $m_{\mathrm{a}}=0.6\left(10^{7} \mathrm{GeV} / f_{\mathrm{a}}\right) \mathrm{eV}$. Astrophysical and cosmological arguments limit this mass to the range $10^{-6} \mathrm{eV} \lesssim m_{\mathrm{a}} \lesssim 10^{-2} \mathrm{eV}$ (see Turner (1990) and Raffelt (1996) for a complete discussion of such bounds).

Axions couple to photons, electrons and nucleons with strengths that depend on the specific implementation of the Peccei-Quinn symmetry. For instance, in the KSVZ - or hadronic model - axions couple to hadrons and photons only (Kim 1979; Shifman et al. 1980), while in the DFSZ or GUT model (Dine et al. 1981; Zhitnitskii 1980), they also couple to charged leptons. Stars can produce axions in their hot and dense interior in analogy as they release thermal neutrinos (Raffelt 1996). These particles can freely escape from stars and act as a sink of energy. In all the implementations, the degree of interaction between axions and matter/photons depends on the mass, a free parameter that is not fixed by the theory. If this mass is large enough, they can noticeably modify the evolution of stars and reveal their existence. In particular, in the case of white dwarfs, axions can modify the cooling rate. If the DFSZ implementation is adopted, the interaction of axions with electrons is dominant and is controlled by the axion fine constant, $\alpha=g_{\mathrm{ae}}^{2} / 4 \pi$, where $g_{\mathrm{ae}}^{2}$ is the dimensionless Yukawa coupling and is related with the mass of the axion by $g_{\mathrm{ae}}^{2}=2.8 \times 10^{-4} m_{\mathrm{a}} \cos ^{2} \beta$, where $\tan \beta$ is the ratio between the two Higgs-field expectation values and $m_{\mathrm{a}}$ is the mass of the axion in $\mathrm{meV}$.

The first hint that axions could be acting in white dwarf stars was provided by the secular drift of the period of pulsation of G117-B15A (Isern et al. 1992), a DA variable white dwarf. The argument, see Fig. 1 of Isern et al. loc.cit., was that since DA variables are placed in the luminosity domain where the transition from a cooling dominated by neutrino bremsstrahlung, $\dot{\epsilon}_{\nu} \propto T^{7}$, to a cooling dominated by photons, $L_{\nu} \propto T^{2.5}$, axion bremsstrahlung, $\dot{\epsilon}_{\mathrm{a}} \propto T^{4}$, could provide the extra cooling term necessary to account for the secular drift observed in G117-B15A.

The second hint was provided by the white dwarf luminosity function (Isern et al. 2008). As can be seen in Fig. 1, the standard theoretical luminosity functions predict a slight excess of stars in the region $M_{\text {bol }} \sim 8-10$ that can be satisfactorily removed if DFSZ axions with $m_{\mathrm{a}} \cos ^{2} \beta \sim 2-10 \mathrm{meV}$ are considered. These results, however, have to be regarded as qualitative, since the uncertainties plaguing the determination of both the observed and theoretical luminosity functions are still large (Isern et al. 2009; Miller Bertolami et al. 2014).

\section{RESULTS AND CONCLUSIONS}

The luminosity function provided by Rowell \& Hambly (2011) was obtained from a sample of the Super Cosmos Sky Survey (SCSS) containing $\sim 10,000$ brighter than $R \sim 19.75$ mag and with proper motions larger than $\mu \sim 0.05$ arcsec $\mathrm{yr}^{-1}$. This luminosity function is statistically significant in the range $6 \lesssim M_{\text {bol }} \lesssim 15$, but it does not provide enough stars beyond the turnover, which is not an inconvenience since we are limiting ourselves to the interval $6 \lesssim M_{\text {bol }} \lesssim 12-13$. From a subsample of white dwarfs with tangential velocities $V_{\text {tan }}>200 \mathrm{~km} \mathrm{yr}^{-1}$, they obtained a noticeably improved, by comparison with the previous ones, luminosity function of the halo. Finally, using statistical techniques, they were able to provide for the first time the white dwarf luminosity functions for the thin and thick disks. Fig. 4 displays these luminosity functions. The solid black line represents the theoretical luminosity functions we have obtained disregarding axion emission and assuming a constant SFR for both the thin and the thick disks, and a burst of short duration for the halo. In all cases, an age of 13 Gyr has been adopted (notice here that neither the adopted age nor the exact shape of the SFR are relevant as far as we are only interested in the bright branch of the luminosity function). As can be seen, in all three cases the standard model of cooling predicts an excess of white dwarfs in the same region around $M_{\mathrm{bol}} \simeq 10$, thus providing support to the hypothesis that the slight shortage of stars in this region is not a consequence of the adopted SFR, but of the existence of an additional cooling mechanism.

Recently, Munn et al. (2017) have improved the Harris et al. (2006) luminosity function of the disk using a sample that contains 2839 stars in the luminosity range $5.5 \simeq M_{\mathrm{bol}} \simeq 17$ and tangential velocities in the range $40 \leqslant v_{\text {tan }} \leqslant 120 \mathrm{~km} \mathrm{~s}^{-1}$ to avoid contamination from subdwarfs. This luminosity function resolves the peak, since it contains 311 objects with bolometric magnitude $M_{\text {bol }} \geqslant 15$ and improves the precision of the brighter bins. The scale height of white dwarfs was assumed to be constant and equal to 300 pc. They also constructed the luminosity function for the halo taking a sample of white dwarfs with $200 \leqslant v_{\tan } \leqslant 500 \mathrm{~km} \mathrm{~s}^{-1}$. Both luminosity functions are displayed in Fig. 5. As in the previous case, visual inspection suggests a shortage of stars in the region $M_{\mathrm{bol}} \approx 10$ in both halo and disk functions.

In Fig. 4 we compare using blue and red solid lines the 


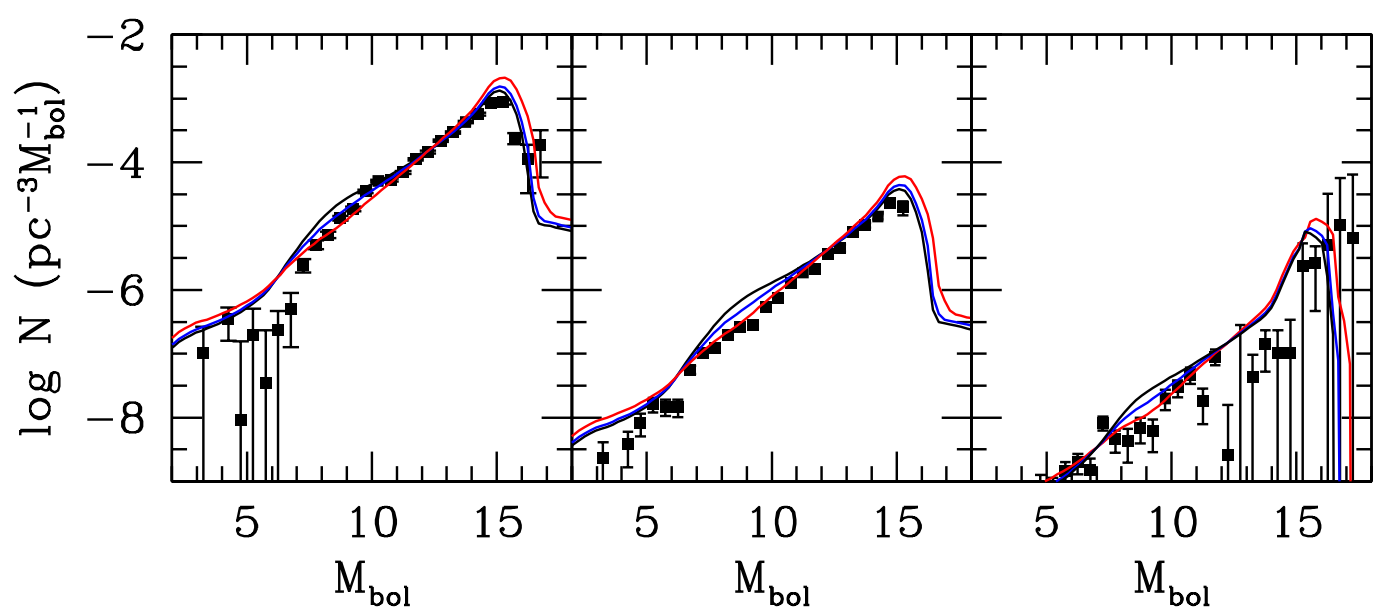

Figure 4. From left to right, luminosity functions of DA and non-Da white dwarfs from the SCSS catalogues for the thin disk, the thick disk and the halo (Rowell \& Hambly 2011). The solid lines represent the luminosity functions obtained disregarding the contribution to cooling of axions (black lines) and considering DFSZ axions with coupling constants $g_{\mathrm{ae}}=2.24 \times 10^{-13}$ and $4.48 \times 10^{-13}$ (blue and red lines, respectively), corresponding to masses $m_{\mathrm{a}} \cos ^{2} \beta \sim 8$ and $16 \mathrm{meV}$.

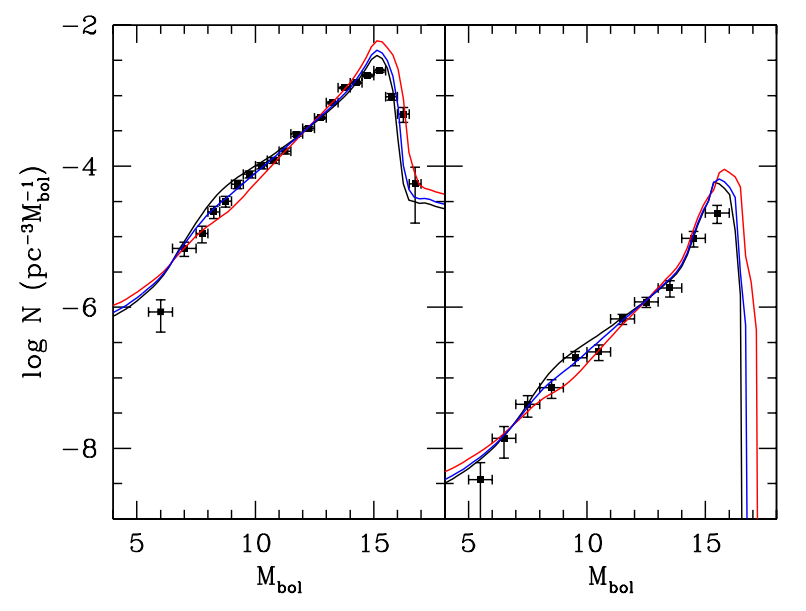

Figure 5. From left to right, luminosity functions of DA and nonDA white dwarfs from the SDSS Munn catalogue for the disk, thin and thick (left panel) and the halo (right panel). The meaning of the solid lines is the same as in Fig. 4.

luminosity functions of the thin and thick disk and halo obtained including DFSZ axions with masses $m_{\mathrm{a}} \cos ^{2} \beta \sim 8$ and $16 \mathrm{meV}$ (equivalent to $g_{a e} \times 10^{13}=2.24$ and 4.48 ) respectively, ${ }^{4}$ with those obtained by Rowell \& Hambly (2011). As can be seen in Table 1, the discrepancies between the theoretical calculations and observations decrease in all three cases, if axions are included. In a similar way,

\footnotetext{
4 The errors used in the calculation of $\chi^{2}$ in Table 1 are just the Poissonian errors. The systematic theoretical and observational uncertainties are larger and not well identified and therefore, they have been excluded. For this reason, we have always claimed that these luminosity functions provide a hint about the existence of axions, not a compelling evidence. The values of $\chi^{2}$ are used here only to illustrate a tendency.
}

Table 1. Reduced $\chi^{2}$ obtained from the comparison between theoretical and observed white dwarf luminosity functions in the brightness interval $6 \lesssim M_{\mathrm{bol}} \lesssim 12.5$ for different intensities of the coupling between electrons and axions.

\begin{tabular}{lcccc}
\hline$g_{\mathrm{ae}} \times 10^{13}$ & 0.00 & 1.12 & 2.24 & 4.48 \\
$m_{\mathrm{a}} \cos \beta(\mathrm{meV})$ & 0 & 4 & 8 & 16 \\
\hline Rowell \& Hambly (2011) & & & & \\
\hline thin disk & 18.59 & 15.33 & 7.33 & 21.52 \\
thick disk & 30.72 & 26.61 & 11.73 & 1.43 \\
halo & 3.31 & 2.94 & 2.36 & 1.87 \\
\hline Munn et al. (2017) & & & & \\
\hline thin+thick disk & 4.89 & 2.87 & 1.46 & 6.13 \\
halo & 2.46 & 1.50 & 0.65 & 1.42 \\
\hline Kilic et al. (2017) & & & & \\
\hline$\Phi_{200-900}$ & 3.25 & 2.09 & 1.05 & 6.11 \\
$\Phi_{200-700}$ & 3.53 & 2.32 & 1.14 & 5.80 \\
$\Phi_{200-500}$ & 4.02 & 2.73 & 1.28 & 2.26 \\
$\Phi_{300}$ & 6.11 & 4.95 & 2.26 & 4.98 \\
\hline
\end{tabular}

Fig. 5 and Table 1 show that in the case of the luminosity functions obtained by Munn et al. (2017), the inclusion of axions of moderate mass improves the concordance between the theory and observations in both, disk and halo.

As we have already mentioned, an important point is the influence of the assumed scale height on the bright branch of the luminosity function. Kilic et al. (2017) constructed several observational luminosity functions with assuming variable scale heights and concluded that the existing slight discrepancy in the region $6 \lesssim M_{\text {bol }} \lesssim 12.5$ is caused by the use of a fixed scale height. In Fig.6 we have repeated the exercise of fitting their functions with our theoretical predictions with and without axions. Once more we have found, see Table 1, that the inclusion of axions improves the agreement between theory and observations. 


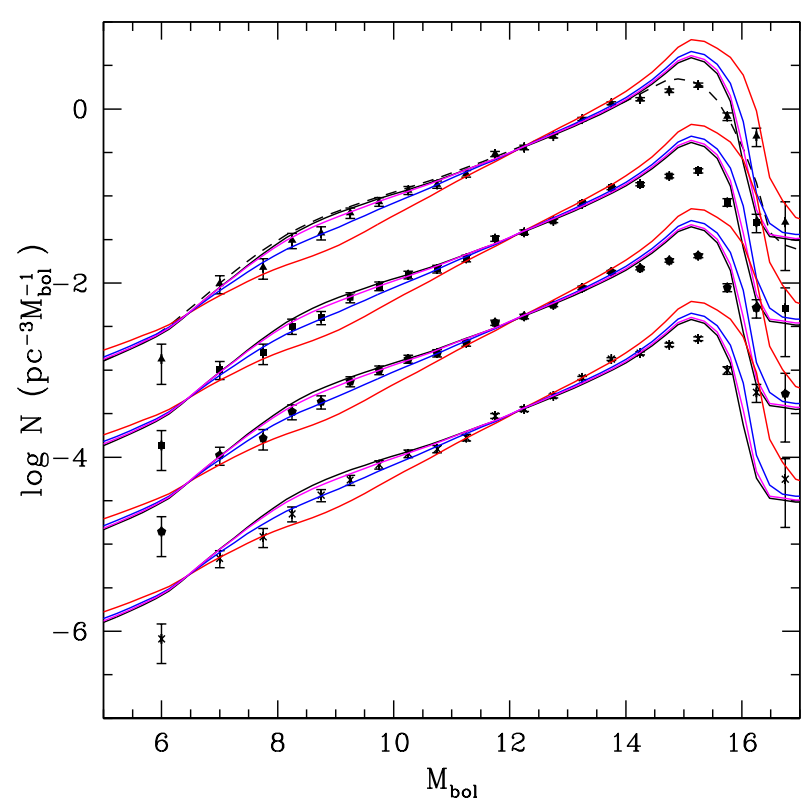

Figure 6. White dwarf luminosity functions of the disk assuming different scale heights as proposed by Kilic et al. (2017). From top to bottom: $\Phi_{200-900}+3$ (triangles); $\Phi_{200-700}+2$ (squares); $\Phi_{200-500}+1$ (pentagons); $\Phi_{300}$ (crosses). The solid lines represent the luminosity functions obtained assuming a constant SFR, no axion cooling (black lines) and DFSZ axion cooling with coupling constants $g_{\mathrm{ae}} \times 10^{13}=1.12,2.24,4.48$ (magenta, blue and red lines, respectively). The dashed line, on the top, represents a case with a constant SFR with an exponential tail and no axions.

All these luminosity functions have been obtained assuming a constant SFR and an age of the disk of 13 Gyr, which is obviously too large. However, as we have shown before, the luminosity function in the region we are considering is not strongly dependent on the age, provided it is large enough, nor on the shape of the old past SFR. As an example, in the top of Fig.6 we have plotted, with black dashed line, the luminosity function corresponding to $\Psi \propto\left(1+\exp \left[\left(t-t_{0}\right) / \tau\right]\right)^{-1}, \tau=3 \mathrm{Gyr}, t_{0}=9 \mathrm{Gyr}$, an SFR that roughly fits the peak of the function.

As can be deduced from Fig. 3, the shape of the luminosity function could be fitted just adding convenient star formation bursts. However, this not seems to be a plausible solution if the star formation history of the halo and discs are uncorrelated or just poorly correlated. Strictly speaking we cannot discard a conspiracy of Nature to produce similar features at the same place in different populations, but it seems more natural to invoke an intrinsic property of white dwarfs. Certainly the different cooling rates of DA and nonDA stars could be a solution but the results obtained in Fig. 2 together with the secular drift of the period of pulsation of ZZ Ceti stars mentioned in Section 2 make the axion hypothesis more attractive. Obviously, these arguments do not prove the existence of axions but make them (axions of DFSZ type with a mass in the range of few meV, 6 to 8 ) a good candidate to account for such a behavior. This possibility could be confirmed in a near future when the new large cosmological surveys (DES, LSST,...), together with the high precision data from the astrometric satellite Gaia will provide accurate and reliable observational white dwarf luminosity functions.

Furthermore, if DFSZ axions exist and have masses in the range of a few $\mathrm{meV}$, as suggested by this study, they will introduce subtle but detectable changes in the evolution of stars as is the case of red giants (Viaux et al. 2013), horizontal branch stars (Ayala et al. 2014), and globular clusters (Hansen et al. 2015; Goldsbury et al. 2012, 2016). Additionally, it is expected that the direct measurement of the characteristic cooling time of white dwarfs employing the secular variation of the pulsation periods of ZZ Ceti stars will be much improved in a near future (Córsico et al. 2012a,b, 2016). This will also allow to obtain more precise estimates of this additional cooling mechanism. Nevertheless, a definitive confirmation of the existence of axions will be provided by the next generation of axioscopes, like IAXO at CERN (Armengaud et al. 2014), that will be able to directly detect the emission of solar axions if their mass, as suggested by the theory of white dwarf cooling, is larger than $3 \mathrm{meV}$.

\section{ACKNOWLEDGEMENTS}

This work has been supported by MINECO grants ESP201566134-R (JI), and AYA2014-59084-P (EG-B), by the European Union FEDER funds, and by grants 2014SGR1458 (JI) and 2014SGR0038 (EG-B) and the CERCA program of the Generalitat de Catalunya. One of us (JI) is very indebted to M. Lam and N. Rowell for the enlightening discussion about the influence of the scale height on the luminosity function.

\section{REFERENCES}

Althaus L. G., Córsico A. H., Isern J., García-Berro E., 2010, A\&ARv, 18, 471

Armengaud E., et al., 2014, Journal of Instrumentation, 9, T05002

Ayala A., Domínguez I., Giannotti M., Mirizzi A., Straniero O., 2014, Physical Review Letters, 113, 191302

Barstow M. A., et al., 2014, preprint, (arXiv:1407.6163)

Catalán S., Isern J., García-Berro E., Ribas I., 2008, MNRAS, 387, 1693

Cignoni M., Degl'Innocenti S., Prada Moroni P. G., Shore S. N., 2006, A\&A, 459, 783

Córsico A. H., Althaus L. G., Romero A. D., Mukadam A. S., García-Berro E., Isern J., Kepler S. O., Corti M. A., 2012a, J. Cosmology Astropart. Phys., 12, 010

Córsico A. H., Althaus L. G., Miller Bertolami M. M., Romero A. D., García-Berro E., Isern J., Kepler S. O., 2012b, MNRAS, 424,2792

Córsico A. H., et al., 2016, J. Cosmology Astropart. Phys., 7, 036 DeGennaro S., von Hippel T., Winget D. E., Kepler S. O., Nitta A., Koester D., Althaus L., 2008, AJ, 135, 1

Dine M., Fischler W., Srednicki M., 1981, Physics Letters B, 104,199

Evans D. W., 1992, MNRAS, 255, 521

Fontaine G., Brassard P., Bergeron P., 2001, PASP, 113, 409

García-Berro E., Oswalt T. D., 2016, New Astron. Rev., 72, 1

Garcia-Berro E., Hernanz M., Isern J., Mochkovitch R., 1995, MNRAS, 277, 801

García-Berro E., Torres S., Althaus L. G., Miller Bertolami M. M., 2014, A\&A, 571, A56

Goldsbury R., et al., 2012, ApJ, 760, 78 
Goldsbury R., Heyl J., Richer H. B., Kalirai J. S., Tremblay P. E., 2016, ApJ, 821, 27

Gratton R. G., Carretta E., Bragaglia A., 2012, A\&ARv, 20, 50

Hansen B., 2004, Phys. Rep., 399, 1

Hansen B. M. S., Liebert J., 2003, ARA\&A, 41, 465

Hansen B. M. S., Richer H., Kalirai J., Goldsbury R., Frewen S., Heyl J., 2015, ApJ, 809, 141

Harris H. C., et al., 2006, AJ, 131, 571

Isern J., García-Berro E., 2008, Mem. Soc. Astron. Italiana, 79,545

Isern J., Hernanz M., Garcia-Berro E., 1992, ApJ, 392, L23

Isern J., García-Berro E., Hernanz M., Mochkovitch R., 1998a, Journal of Physics Condensed Matter, 10, 11263

Isern J., García-Berro E., Hernanz M., Mochkovitch R., Torres S., 1998b, ApJ, 503, 239

Isern J., García-Berro E., Torres S., Catalán S., 2008, ApJ, 682, L109

Isern J., Catalán S., García-Berro E., Torres S., 2009, Journal of Physics Conference Series, 172, 012005

Isern J., Artigas A., García-Berro E., 2013, in European Physical Journal Web of Conferences. p. 05002 (arXiv:1212.0806), doi:10.1051/epjconf/20134305002

Kilic M., Munn J. A., Harris H. C., von Hippel T., Liebert J. W., Williams K. A., Jeffery E., DeGennaro S., 2017, ApJ, 837, 162

Kim J. E., 1979, Physical Review Letters, 43, 103

Kiziltan B., Baumgardt H., Loeb A., 2017, Nature, 542, 203

Knox R. A., Hawkins M. R. S., Hambly N. C., 1999, MNRAS, 306, 736

Koester D., 2002, A\&ARv, 11, 33

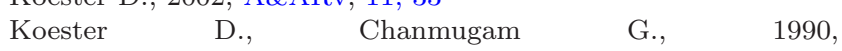
Reports on Progress in Physics, 53, 837

Krzesinski J., Kleinman S. J., Nitta A., Hügelmeyer S., Dreizler S., Liebert J., Harris H., 2009, A\&A, 508, 339

Leggett S. K., Ruiz M. T., Bergeron P., 1998, ApJ, 497, 294

Liebert J., Dahn C. C., Monet D. G., 1988, ApJ, 332, 891

Limoges M.-M., Bergeron P., 2010, ApJ, 714, 1037

Miller Bertolami M. M., Melendez B. E., Althaus L. G., Isern J., 2014, J. Cosmology Astropart. Phys., 10, 069

Munn J. A., et al., 2017, AJ, 153, 10

Noh H.-R., Scalo J., 1990, ApJ, 352, 605

Oswalt T. D., Smith J. A., Wood M. A., Hintzen P., 1996, Nature, 382,692

Peccei R. D., Quinn H. R., 1977a, Phys. Rev. D, 16, 1791

Peccei R. D., Quinn H. R., 1977b, Physical Review Letters, 38,1440

Raffelt G. G., 1996, Stars as laboratories for fundamental physics : the astrophysics of neutrinos, axions, and other weakly interacting particles. University of Chicago Press

Reid I. N., 2005, ARA\&A, 43, 247

Renedo I., Althaus L. G., Miller Bertolami M. M., Romero A. D., Córsico A. H., Rohrmann R. D., García-Berro E., 2010, ApJ, 717,183

Rowell N., 2013, MNRAS, 434, 1549

Rowell N., Hambly N. C., 2011, MNRAS, 417, 93

Salaris M., Cassisi S., Pietrinferni A., Kowalski P. M., Isern J., 2010, ApJ, 716, 1241

Shifman M. A., Vainshtein A. I., Zakharov V. I., 1980, Nuclear Physics B, 163, 46

Turner M. S., 1990, Phys. Rep., 197, 67

Viaux N., Catelan M., Stetson P. B., Raffelt G. G., Redondo J., Valcarce A. A. R., Weiss A., 2013, Physical Review Letters, 111,231301

Weidemann V., 1968, ARA\&A, 6, 351

Weinberg S., 1978, Physical Review Letters, 40, 223

Wilczek F., 1978, Physical Review Letters, 40, 279

Zhitnitskii A. P., 1980, Soviet Journal Nuclear Physics, 31, 260 